\section{An Infant With Isolated Motor Delay}

An 11-month-old male infant, first born of a nonconsanguineous marriage was brought with concerns of delayed motor milestones. He had an uneventful antenatal and perinatal period. He achieved head control at 6 months of age and rolling over at 10 months of age. He had normal social and cognitive milestones. None of the family members in a three-generation pedigree had symptoms suggestive of any neuromuscular illness. On examination, he had peripheral hypotonia, diminished deep tendon reflexes in both upper and lower limbs, without any tongue fasciculation or signs of facial, extraocular, bulbar, and cardiac muscle involvement. Serum creatinine phosphokinase was found to be elevated (2568 IU/L). A clinical possibility of Pompe disease, congenital muscular dystrophies and congenital myopathies (central core and multiminicore myopathy) was considered. Muscular dystrophy and congenital myopathy genetic panel revealed a hemizygous pathogenic nonsense variation in exon 61 of the $D M D$ gene (ChrX:g:31366736G>A), confirming a diagnosis of Duchenne muscular dystrophy (DMD). The observed variation was confirmed by sanger sequencing. It found to be previously reported in patients with DMD and has been classified as pathogenic in ClinVar database. The in silico prediction of the variant was damaging by Mutation Taster 2. He was started on oral prednisolone $(0.3 \mathrm{mg} / \mathrm{kg} / \mathrm{day})$ and physiotherapy and parents were counseled about the nature and prognosis of the disease.

Duchenne muscular dystrophy (DMD) is the commonest muscular dystrophy having an incidence rate of one in every 3500 male infants [1]. Indian data suggests that exonic deletions and duplications are found in around $67 \%$ and $6 \%$ boys with DMD, respectively. Most of the cases become symptomatic between 2 to 6 years of age, with frequent falls during walking, difficulty in getting up from sitting or squatting position, and waddling gait [1]. However, few recent studies have revealed that a proportion of children with DMD had a delay in the attainment of motor milestones from infancy [2]. Although some of these parents often express the developmental concern of their children in toddler years, differential diagnosis of DMD is rarely considered in these children because of the absence of muscle weakness [2]. In the existing literature, the youngest age at which diagnosis was established in symptomatic DMD cases was 3 years of age, although new-born screening and screening of affected siblings have detected asymptomatic cases in infants
[2]. The common causes of peripheral hypotonia with elevated CPK levels in infants include congenital muscular dystrophy (CMD), congenital myopathies (central core and multiminicore myopathy), and metabolic myopathies like Pompe's disease [3]. Children with congenital muscular dystrophy usually have much higher serum CPK levels with hypotonia, while children with secondary merosin deficient CMD often have epilepsy, cognitive impairment to some extent and brain malformations. Children with congenital myopathies have predominantly ocular, facial and bulbar involvement along with mildly elevated serum CPK. Infants with Pompe disease have hepatomegaly and cardiomyopathy along with peripheral hypotonia and elevated CPK levels. Infantile polymyositis is another rare possibility in such cases, which unlike older children, may sometimes present with isolated motor delay and elevated serum CPK without any fever or systemic features.

Early diagnosis of DMD often provides an opportunity for timely institution of treatment including drugs like steroids, ataluren and eteplirsen, physiotherapy and genetic counselling of parents for subsequent conceptions [4]. Early institution of glucocorticoids in low doses, as soon as the diagnosis is established, has been shown to improve the outcome at the cost of tolerable side effects, although not able to cure the disease. Glucocorticoids were initiated in the index case after discussing risks and benefits with parents.

To conclude, while evaluating an infant with raised CPK levels, clinicians should consider DMD as one of the differential diagnosis apart from CMD and few selected congenital myopathies. Early diagnosis and initiation of steroids may improve the outcome at the cost of tolerable side effects.

Indar Kumar Sharawat and Prateek Kumar Panda* Pediatric Neurology Division, Department of Pediatrics, All India Institute of Medical Sciences, Rishikesh, Uttarakhand. India. *drprateekpanda@gmail.com

\section{REFERENCES}

1. Thangarajh M. The Dystrophinopathies. Contin Minneap Minn. 2019;25:1619-39.

2. Tallapaka K, Ranganath P, Ramachandran A, Uppin MS, Perala S, Aggarwal S, et al. Molecular and histopathological characterization of patients presenting with the duchenne muscular dystrophy phenotype in a tertiary care center in Southern India. Indian Pediatr. 2019;56:556-59.

3. Leyenaar J, Camfield P, Camfield C. A schematic approach to hypotonia in infancy. Paediatr Child Health. 2005;10:397400.

4. Mah JK. Current and emerging treatment strategies for Duchenne muscular dystrophy. Neuropsychiatr Dis Treat. 2016;12:1795-8. 